Cita: Silva, C; Rosado, A. (2019). Efeitos psicossociais do Yoga: ouvir as vozes de professores de Yoga portugueses experientes. Cuadernos de Psicología del Deporte, Vol 19(1), 121-146

\title{
Efeitos psicossociais do Yoga: ouvir as vozes de professores de Yoga portugueses experientes
}

\section{Los efectos psicosociales del Yoga: escuchar las voces de experimentados profesores portugués de Yoga}

\section{Yoga and Psychosocial effects: listen to the voices of Portuguese Experienced Yoga Teachers}

\author{
Silva, Catarina Pissarra ${ }^{1}$, Rosado, António Boleto ${ }^{1}$ \\ ${ }^{1}$ Faculty of Human Kinetics, University of Lisbon, Portugal
}

\section{RESUMO}

A maioria da investigação sobre os efeitos da prática do Yoga centra-se em estudos empíricos da área médica sendo escassos os estudos centrados nos seus efeitos psicossociais. Este estudo pretende conhecer as opiniões de professores experientes de Yoga, portugueses, com muita experiência de prática e lecionação de Yoga, sobre os efeitos psicossociais da prática do Yoga. Pretende-se conhecer em que medida o Yoga influenciou as suas vidas e relatar testemunhos que evidenciem a sua perceção.

Utilizou-se uma metodologia qualitativa, com entrevistas semiestruturadas a dez professores, cinco de cada sexo, com idades entre os 40 e os 95 anos, dois com mais de 90 anos, todos com muita experiência prática $\left(M_{\text {anos }=} 45,8\right.$ e $\left.D P=16,47\right)$ e de lecionação $\left(M_{\text {anos }}=34,2\right.$ e $\left.D P=13,48\right)$. As entrevistas, individuais, foram gravadas e transcritas integralmente. O conteúdo dos dados foi analisado com o Programa ATLAS. ti 7 e realizou-se uma Análise Temática, com a divisão dos conteúdos em temas e categorias emergentes.

Os resultados indicam que todos os professores consideram que a prática de Yoga tem efeitos psicossociais positivos, nomeadamente, a nível da melhoria do bem-estar físico e social, do bem-estar espiritual, do otimismo, da satisfação com a vida, da felicidade, da resiliência, da atenção plena e das autoavaliações nucleares.

Palavras chave: Bem-estar; otimismo; satisfação com a vida; resiliência; atenção plena.

\section{RESUMEN}

La mayoría de las investigaciones sobre los efectos de la práctica del Yoga se centra en estudios empíricos de área médica y pocos estudios centrados en sus efectos psicosociales. Este estudio pretende conocer las opiniones de experimentados profesores de Yoga, portugués, con mucha experiencia en la práctica y enseñanza de Yoga, sobre los efectos psicosociales de la práctica del Yoga. Desea saber en qué medida el Yoga ha influido en sus vidas y testimonios de informes que prueben sus percepciones. Se utilizó una metodología cualitativa, entrevistas estructuradas con diez profesores, cinco de cada sexo, con 


\section{Silva, Catarina, Rosado, António}

edades entre 40 y 95 años, dos de ellos con más de 90 años, todos con mucha experiencia práctica $(M=$ $45,8$ y $D T=16.47)$ y lecionação $(M=34,2$ y $D T=13.48)$. Las entrevistas individuales fueron grabadas y transcritas en su totalidad. El contenido de los datos se analizaron con el programa AtlasTi7 y habiendo realizado un análisis temático, con la división del contenido en categorías y temas emergentes. Los resultados indican que todos los profesores creen que la práctica de Yoga tiene efectos psicosociales positivos, en particular, en el nivel de mejora del bienestar físico y social, bienestar espiritual, el optimismo, la satisfacción con la vida, la felicidad, la resiliencia le mindfulness y las auto-evaluaciones nucleares.

Palabras clave: Bienestar, optimismo, life satisfacción con la vida, resiliencia, mindfulness.

\section{ABSTRACT}

Most of the research on the effects of Yoga practice focuses on empirical studies in the medical field, with few studies focusing on the psychosocial effects of its practice, with emphasis on the relationship between the practice of Yoga and the constructs of positive psychology. This study intends to know the opinions of expert teachers of Yoga, Portuguese, with much experience of practice and Yoga teaching, about the psychosocial effects of Yoga practice. To know also if Yoga influenced their lives and to report testimonies that evidences their perception of the psychosocial effects of this practice.

A qualitative methodology was used, whit semi-structured interviews with ten teachers, Yoga experts, five females and five males, aged between 40 and 95 years, two over 90 years old, all with a lot of practical experience $(M=45.8$ and $S D=16.47)$ and teaching $(M=34.2$ and $S D=13.48)$ teaching in Portugal. The individual interviews are transcribed in full. The content of the data was analyzed with Atlasti7 program and a thematic analysis was carried out, with the division of contents into emerging themes and categories. The results indicate that all teachers participating in the study consider that the practice of Yoga has positive psychosocial effects, namely, in the improvement of physical and social well-being, spiritual well-being, optimism, satisfaction with life, happiness, resilience, mindfulness and nuclear self-evaluations.

Keywords: Well-being, optimism, life satisfaction, resilience, mindfulness.

\section{INTRODUÇÃO}

O interesse pelo Yoga tem crescido nos anos recentes, assim como tem aumentado o seu número de praticantes. Existem evidências de que a prática de Yoga promove efeitos positivos a nível físico e psíquico, com uma melhoria do bem-estar dos praticantes. No entanto, a quase totalidade dos estudos foca-se na identificação dos efeitos do Yoga na saúde, referindo benefícios em diversas patologias, verificando-se existir uma melhoria a nível da saúde e da qualidade de vida nos indivíduos que praticam Yoga (Silva e Rosado, 2017). Estes autores indicam que, em populações diversificadas e relativamente a diferentes construtos psicossociais, a prática de Yoga tem efeitos positivos, nomeadamente ao nível da perceção do bem-estar (Bilderbeck, Farias, Brazil, 


\section{Efeitos psicossociais do Yoga}

Jakobowitz, e Wikholm, 2013; Moliver, Mika, Chartrand, Houssman, e Khalsa, 2013; Rathore, e Choudhary, 2013; Reis e Alligood, 2014; Rissel, Miller, Lloyd, e Williams, 2014; Taspinar, Aslan, Agbuga, 2014, e Telles, Singh, Bhardwaj, Kumar, e Baikrishna, 2013). A resiliência é abordada em outros estudos empíricos, aparecendo como sendo, também, influenciada pela prática de Yoga (Ivtzan e Papantoniou, 2014; Telles, Singh, Bhardwaj, Kamar, e Baikrishna, 2013). Outros estudos verificaram efeitos na ampliação do otimismo (Reis e Alligood, 2014), uma maior satisfação com a vida (Ivtzan e Papantoniou, 2014; Rissel, Miller, Lloyd, e Williams, 2014); e melhoria do bem-estar espiritual (Ivtzan e Papantoniou, 2014; Rathore e Choudhary, 2013; Reis e Alligood, 2014). Também as autoavaliações nucleares são influenciadas positivamente com a prática de Yoga (Rissel, Miller, Lloyd, e Williams, 2014).

Os mesmos autores alegam que a qualidade da investigação produzida, na maioria das revisões sistemáticas e artigos empíricos sobre efeitos do Yoga, apresenta algumas lacunas metodológicas. Identificaram problemas nos processos de seleção e dimensão das amostras, no delineamento dos estudos, no facto de não serem devidamente controladas as ameaças à validade interna e externa. Relativamente às intervenções realizadas, muitas vezes, estas não se encontram suficientemente detalhadas e decorrem num período de tempo demasiado curto. Todas estas limitações contribuem para diminuir a possibilidade de retirar conclusões sobre os reais efeitos da prática de Yoga.

Groessi, Chopra, e Mills (2015) apresentaram uma revisão da investigação sobre o impacto da prática do Yoga a nível da saúde e do bem-estar. Estes consideraram a saúde de uma forma holística, na tradição yoguica, com áreas específicas interligadas como a saúde física, mental, e o bem-estar espiritual. Concluíram que a prática de Yoga tem efeitos positivos e replicáveis em muitas condições de saúde.

Recentemente, Halsall, Werthner, e Forneris (2015) concluíram, num estudo com entrevistas a praticantes, que a prática de Yoga longa e consistente no tempo contribui para um aumento da resiliência, com um impacto positivo no bemestar. Casaban, Bix, Delcmpo, e Chirivella (2017) num estudo com uma intervenção em que foram utilizadas técnicas de relaxação verificaram um efeito positivo nalgumas dimensões do bem-estar psicológico e na autoestima. Numa intervenção com atletas olímpicos, Spaccartella (2017) utilizou também sessões de relaxação e acrescentou visualizações para potenciar a relaxação física e as emoções positivas e obteve resultados positivos.

$\mathrm{Na}$ validação de uma escala sobre o Fluxo Disposicional, Gouveia, Pais-Ribeiro, Marques, e Carvalho (2012) referem que na prática de 


\section{Silva, Catarina, Rosado, António}

exercícios de movimento meditativo como o Yoga, o foco na atenção, no momento e naquilo que está a acontecer é valorizado por estas práticas sugerindo a necessidade de estudos deste tipo de actividade física no bem-estar.

O presente estudo tem por objectivo estudar os efeitos psicossociais do Yoga na perspetiva de professores experts em Portugal, com muitos anos de experiência de lecionação e oriundos de escolas diferentes. Pretende-se identificar os efeitos que reconhecem como fruto da sua prática, referindo como o Yoga contribuiu para as suas vidas, e os efeitos da prática nos seus alunos, indicando se a prática pode melhorar diversos construtos psicossociais pouco estudados, nomeadamente, a nível do bem-estar, do bem-estar espiritual, do otimismo, da satisfação com a vida, da felicidade, da resiliência, da atenção plena e das autoavaliações nucleares.

\section{MATERIAL E MÉTODOS}

A investigação qualitativa baseia-se no paradigma construtivista que considera a existência de realidades múltiplas e que estas são, segundo Maxweel (2013), uma construção social. Neste tipo de pesquisa o investigador, como instrumento de investigação representa um papel fundamental na forma como os dados são recolhidos e analisados.
$\mathrm{Na}$ investigação qualitativa as entrevistas podem ser usadas para examinar as atitudes, crenças e valores dos participantes em relação às suas experiências, contribuindo para uma maior compreensão do comportamento humano. A metodologia qualitativa deve, segundo Lolina, Villanón e Úbeda (2015), ser promovida porque permite ampliar o campo de investigação nas ciências da atividade física.

A abordagem qualitativa oferece uma grande flexibilidade, mas a interpretação do contexto e da forma apresenta de acordo com Anguera, Portell, Chacon-Moscoso, e Sandavete-Chaves (2018) alguma controvérsia.

\section{Participantes}

Os participantes foram selecionados seguindo os seguintes critérios: (i) professores de Yoga conhecidos na comunidade de Yoga como pioneiros em Portugal, (ii) reconhecidos como especialistas, (iii) com conhecimento aprofundado da temática, (iv) com dez ou mais anos de prática e lecionação de Yoga. O tempo de lecionação e de prática está de acordo com as indicações de Ericson, Krampe, e Tesch-Romer (1993) que consideram que para um desempenho experiente é necessária uma prática intensa e deliberada de pelo menos dez anos.

A amostra é intencional, inserindo-se na subcategoria de amostra de intensidade. Neste tipo de amostra a estratégia, de acordo com 


\section{Efeitos psicossociais do Yoga}

Patton (2002), passa por envolver uma selecção de casos que proporcionem um conhecimento aprofundado sobre o fenómeno abordado mas que não sejam casos incomuns. No presente estudo, o processo de amostragem baseou-se, ainda, num processo de "bola de neve", que permite aceder a populações com baixa incidência. Os professores participantes indicaram outros experts da sua rede pessoal que foram posteriormente contactados.

O número de entrevistados está de acordo com Saunders et al. (2017) que consideram que deve existir flexibilidade relativamente ao momento em que se considera ter atingido a saturação quando se realizam entrevistas sobre uma determinada temática, o que depende do tipo de estudo, bem como do que pode ser considerado diferente ou novo sobre o objeto de estudo. A saturação aconteceu quando as opiniões se começam a repetir, não surgindo novos dados que acrescentem algo ao tema abordado. As entrevistas terminaram quando começaram a ser confirmadas as afirmações, não existindo mais contribuições para um melhor entendimento concetual.

Assim, participaram 10 professores, cinco de cada sexo, com idades entre os 40 e 95 anos, dois dos professores com idade superior a 90 anos, um destes leciona, ainda, diariamente. $\mathrm{O}$ número de anos de prática situa-se entre 16 e 70 anos $\left(M_{\text {anos }}=43,3, D P=16,47\right)$ e de lecionação entre 14 e $\quad 60$ anos $\left(M_{\text {anos }}=34,7, \quad D P=13,48\right)$.

Relativamente à sua formação os professores indicaram que realizaram formação inicial especializada em Yoga em Centros Internacionais e continuaram com a sua formação de forma regular e contínua para o seu aperfeiçoamento. Todos foram ou continuam a ser formadores de professores de Yoga. Para cada participante, apresenta-se um quadro com a sua apresentação, com indicação do sexo, da idade, dos anos de prática e de lecionação e, transcreve-se uma frase que caracteriza, de forma sucinta, a sua relação com o Yoga (Tabela 1).

Tabela 1. Caraterização dos entrevistados e transcrição breve do que é o Yoga para os entrevistados

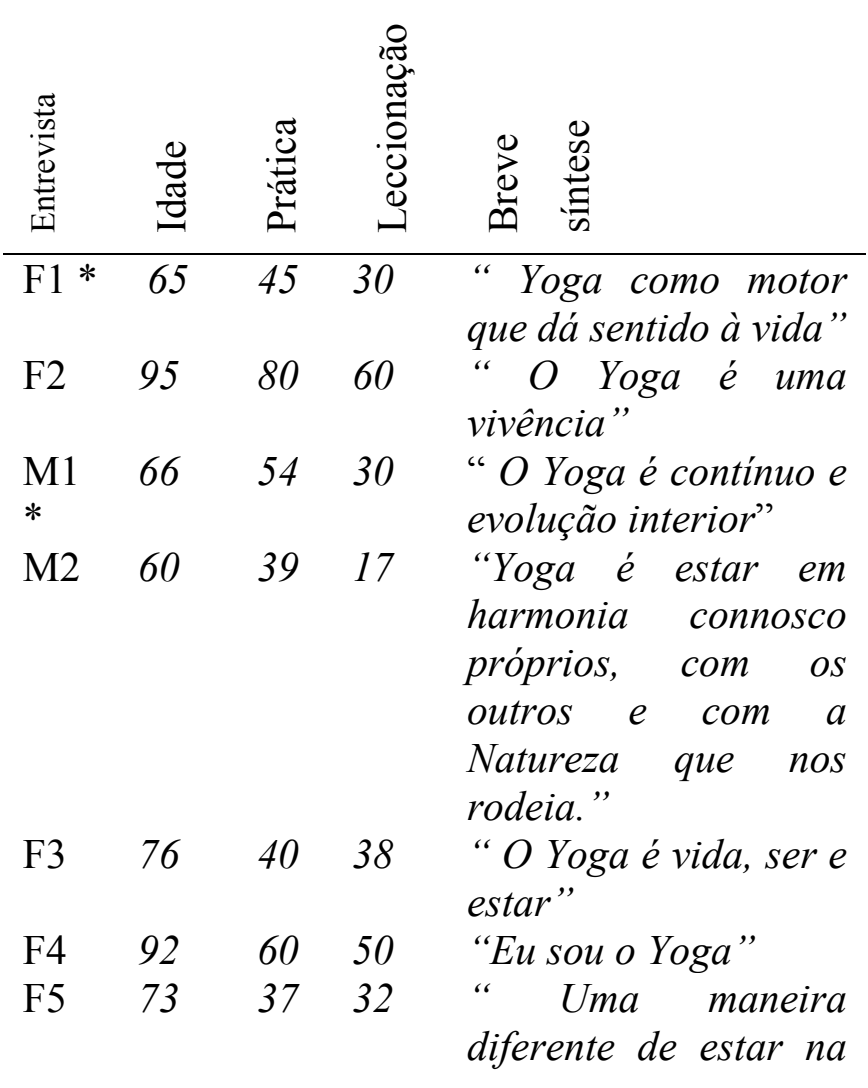




\section{Silva, Catarina, Rosado, António}

\begin{tabular}{|c|c|c|c|c|}
\hline & & & & vida" \\
\hline M3 & 63 & 43 & 35 & $\begin{array}{l}\text { "O Yoga é o meu } \\
\text { alento, o meu canto } e \\
\text { encanto" }\end{array}$ \\
\hline M4 & 54 & 43 & 35 & $\begin{array}{l}\text { "O Yoga é ampliar a } \\
\text { consciência" }\end{array}$ \\
\hline M5 & 40 & 17 & 15 & $\begin{array}{l}\text { "O Yoga, a união com } \\
o \text { doce mistério, é } \\
\text { claramente a direção } \\
\text { da minha existência" }\end{array}$ \\
\hline
\end{tabular}

Notas: * F (sexo feminino) e M (sexo masculino), prática em anos e lecionação em anos

\section{Instrumentos}

A entrevista semiestruturada foi escolhida porque permite flexibilidade na conversação, alargando o espetro da entrevista, permitindo o aprofundamento e a emergência de novos conceitos no diálogo entre os participantes e o investigador (Lincoln e Guba, 1985). O protocolo da entrevista foi submetido a validação por peritagem de três juízes e, de seguida, aplicado a dois professores num estudo piloto. Estas entrevistas iniciais contribuíram para desenvolver o guião final de entrevista $\mathrm{e}$ determinar estratégias para conseguir revelar melhor o conhecimento dos participantes. Foram revistos o estilo de interação, a utilização de questões, o controlo do tempo, o registo audio e a eficácia do guião.

As entrevistas tiveram uma duração entre 45 e 71 minutos $\left(M_{\text {duração= }} 50,2\right.$ minutos $)$, todas gravadas e transcritas integralmente.

\section{Procedimento}

Neste estudo conduziram-se entrevistas em profundidade, com uma parte inicial de caracterização demográfica, após o que os professores foram convidados a explorar temas específicos.

A pesquisa tem uma perspectiva de análise temática seguindo-se as indicações de Braun e Clarke (2006). Neste estudo, concomitantemente às entrevistas, 0 investigador tomou notas destacando as impressões que acompanharam a recolha de dados, sendo essas notas integradas na análise das temáticas, após as transcrições e nos códigos preliminares dos significados subjacentes. Em seguida, os autores reviram os códigos organizando os dados hierarquicamente em temas e subtemas. Os temas que emergiram esclarecem as similaridades comuns aos participantes bem como as suas experiências únicas.

Procurando-se, ainda, perceber de forma mais aprofundada, a experiência vivida na perspetiva dos participantes, são apresentados extractos das entrevistas que permitem perceber a essência do que se pretende demonstrar, de acordo com Braun e Clarke (2006).

Seguem-se, ainda, as indicações de Tracy (2010), que considera que, no delineamento de um estudo, deve verificar-se um tópico rigoroso, sinceridade, credibilidade, ressonância, coerência significativa. 


\section{Efeitos psicossociais do Yoga}

Para garantir a credibilidade da investigação, as entrevistas foram transcritas integralmente, tendo-se solicitado aos participantes que revissem a transcrição da informação transcrita, confirmando a fidelidade dos registos (member cheking) (Meadows e Morse, 2001). Verificouse, ainda, que a experiência como praticante e professor de Yoga do primeiro investigador facilitou a preparação para a recolha de dados, o acesso aos participantes e melhorou a relação com os entrevistados. A ressonância é demonstrada através de descrições vividas nas narrativas dos participantes.

Explora-se, também, a apresentação visual da informação recolhida, com o programa WordArt. O estudo foi aprovado pelo Conselho de Ética da Faculdade de Motricidade Humana (Parecer 11/2017). Toda a informação identificativa foi removida e os procedimentos utilizados foram objeto de consentimento informado de todos os participantes possibilitando a realização do estudo.

\section{Análise estatística}

As etapas seguidas na análise de dados seguiram as indicações de Pérez-Lopez, Morales-Sanchez, Anguerra, e Hernández-Mendo (2014). Os dados foram sujeitos a exame indutivo e dedutivo através de um processo de análise temática. $\mathrm{O}$ processo compreendeu uma codificação indutiva inicial dos dados envolvendo o escrutínio line- by-line do que foi dito pelo entrevistado (Lincoln, e Guba, 1985). O texto foi segmentado em unidades de texto. Isso ajudou a separar os dados em categorias, oferecendo mais pistas para prosseguir. As categorias, mutuamente exclusivas, com conteúdos próximos, tiveram como base a estrutura teórica, o seu núcleo categorial, ou seja, o conteúdo de cada categoria que a diferencia das outras baseia-se na teoria e os núcleos categoriais deram lugar ao sistema de categorias. Estas ligações foram seguidas através de um processo semelhante a uma codificação mais focada, que resultou em códigos cada vez mais seletivos e diretos, como apontam Braun e Clarke (2006). Criaram-se famílias de códigos agrupando códigos relacionados. Elaboraram-se gráficos que representam relações entre códigos. Utilizou-se como suporte o Programa de Análise Qualitativa de Dados ATLAS.ti 7 (Friese, 2013), para segmentar e codificar o texto. O referido programa é reconhecido na análise qualitativa como indicam Pérez-López, Morales-Sanchez, Anguera, e Heranndez-Mendo (2016). Os mesmos autores, Pèrez-López, MoralesSanchez, Angera, e Hernadez-Mendo (2015), indicam, como principais vantagens deste programa, a facilidade de transmitir uma ideia, ajudando no trabalho concetual e na representação gráfica dos códigos e das suas relações, auxiliando o trabalho de análise, interpretação e explicação dos resultados. 


\section{Silva, Catarina, Rosado, António}

A codificação foi revista por um especialista para validar os processos de análise dos dados qualitativos determinando-se os totais de acordos e de desacordos entre os investigadores e o especialista. Aplicando-se a fórmula de Amado (2014), o valor encontrado foi de $r=0,77$.

Os documentos primários, constituídos pelas transcrições das entrevistas, foram organizados e armazenados num arquivo único no programa ATLAS.ti 7 onde se encontra toda a informação, os códigos e famílias de códigos. Apresentam-se figuras retiradas a partir do referido programa.

\section{RESULTADOS}

Cada documento primário corresponde a cada uma das entrevistas, os códigos são os elementos principais de análise, ou seja, as categorias, encontrando-se agrupados em famílias permitindo a redução dos dados. $\mathrm{Na}$ Tabela 2 encontram-se a totalidade dos códigos com o respetivo número de citações na totalidade das entrevistas

Tabela 2. Códigos com número de citações (número

\begin{tabular}{|c|c|c|c|}
\hline Anos lecionação \{10 & Efeitos psicológicos \{18 & Meditação \{37 & 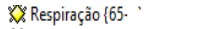 \\
\hline Anos prática $\{10$ & Escolaridade $\{5$. & Organizaçōes \{1 & Satisfação com a vida $\{13\}$ \\
\hline * Atenção plena $\{25$ & Estabilidade emocional \{14 & Otimismo $\{11$. & 次 Saúde \{16 \\
\hline 永Aulas \{38 & Felicidade $\{18$. & 2 Posturas $\{51$. & 發 uniâo $\{0-$ \\
\hline Auto-avaliaçōes nucleares (32 & Formação de professores \{17 & Problemas $\{21$ & Vida pessoal \{38- \\
\hline Bem estar $\{23$ & 20ormação ética \{15. & 㟋 Profissão \{4. & Zogoterapia \{14. \\
\hline Bem estar espiritual \{18 & Formação pessoal \{22 & Psíquicos $\{0$ & \\
\hline Benefícios académicos $\{24$ & * Fruição estética \{1. & Qualidade de vida \{21 & \\
\hline 㟋 Destacar $\{35$ & Género \{1. & Rendimento Desportivo $\{12$. & \\
\hline Duas palavras $\{14$ & Hábitos alınentares $\{17$ & Resiliência \{28 & \\
\hline Efeitos Fisicos \{27 & 淡 Idade $\{9$ - & Resistência mental \{18 & \\
\hline 敢 Efeitos Mentais \{22 & 泳 Início prática \{13. & Resolução de problemas $\{0$ - & \\
\hline Efeitos negativos \{20 & 永 Mantras \{3. & Respeito pela natureza $\{1$ & \\
\hline
\end{tabular}

Os códigos de família agrupam códigos como se verifica na Figura 1, em que a família dos efeitos psicossociais, tem associados seguintes os códigos, com indicação do número de vezes em que são citados na totalidade das entrevistas: autoavaliações nucleares, felicidade, satisfação com a vida, otimismo, resiliência, atenção plena, bem-estar, resistência mental e estabilidade emocional. (por exemplo o código Bem-estar surge na totalidade das entrevistas referenciado

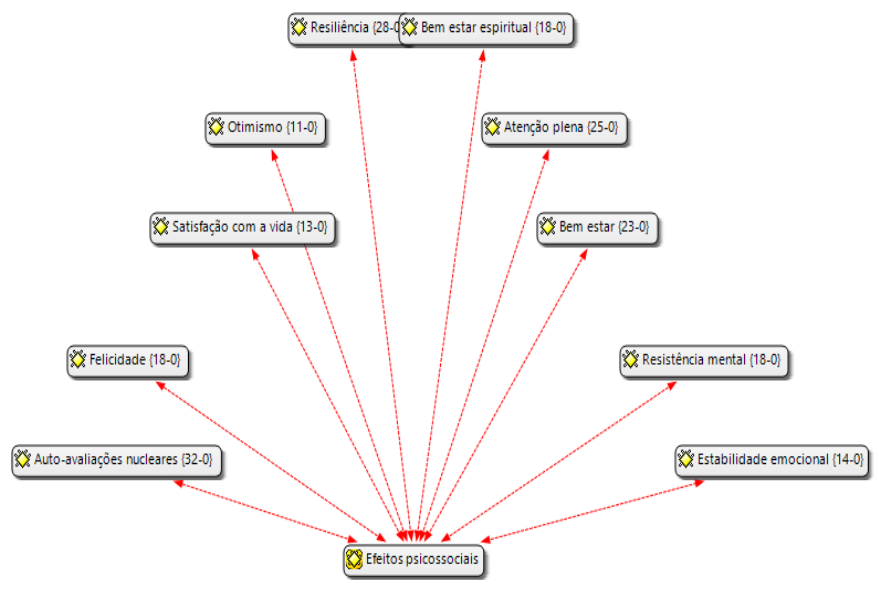

23 vezes).

Legenda: categorias e número de citações

Figura 1. Família de Códigos em árvore, efeitos psicossociais, com indicação do número de citações.

Na Tabela 3 apresenta-se o número de vezes que cada código apresenta, com o correspondente número de citações. A família código efeitos psicossociais apresenta o número do somatório de todas as citações referenciando os efeitos nos vários construtos psicossociais. 


\section{Efeitos psicossociais do Yoga}

Tabela 3. Relação da frequência de categorias e número de citações.

\begin{tabular}{|c|c|}
\hline Name & Size \\
\hline 总Atenção plena & 26 \\
\hline 品 Auto-avaliações nuclea & 33 \\
\hline 遂 Bem estar & 24 \\
\hline 品 Bem estar espiritual & 19 \\
\hline 最Efeitos psicossociais & 11 \\
\hline 吕Felicidade & 19 \\
\hline 品Otimismo & 12 \\
\hline 足 Resiliência & 29 \\
\hline 苑 Satisfação com a vida & 14 \\
\hline
\end{tabular}

Legenda: name- categorias e size - número de citações

Realizou-se, ainda, uma análise de coocorrências na Tabela 4, em que na forma de uma tabela, se apresenta a co-ocorrência das categorias, ou seja, as categorias mais relacionadas com cotações similares. Existe uma co-ocorrência quando é codificado um segmento da entrevista, no todo ou em parte, com mais do que uma categoria. Na figura quando não existe co-ocorrência a célula não se encontra preenchida e quando existe a célula apresenta o número de vezes em que se verifica a coocorrência e em seguida o c-coeficiente, este indica a força da relação entre duas categorias, mas este valor só é significativo numa amostra de tamanho razoável, o que não se verifica, de acordo com as instruções do Programa ATLAS.ti 7, numa amostra com 10 entrevistados. A intensidade da tonalidade no preenchimento das células indica a força da relação entre as categorias, sendo mais intensa quando mais clara.

Verifica-se a existência de co-ocorrências entre vários pares de categorias: (i) entre autoavaliações nucleares e a resiliência, a atenção plena, o bem-estar, a felicidade, a satisfação com a vida e o otimismo, (ii) entre a resiliência, a atenção plena, o bem-estar, o bemestar espiritual e a satisfação com a vida, (iii) entre a atenção plena, o bem-estar espiritual e a felicidade, (iv) entre o bem-estar,o bem-estar espiritual, a satisfação com a vida e o otimismo, (v) entre o bem-estar espiritual e a felicidade, (vi) entre a felicidade, a satisfação com a vida e o otimismo, (vii) entre a satisfação com a vida e o otimismo.

Tabela 4. Tabela de co-ocorrência de códigos

\begin{tabular}{|c|c|c|c|c|c|c|c|c|}
\hline \multirow[b]{2}{*}{ Auto-svalisçọes nucleares } & \multicolumn{2}{|c|}{ Auto-svalisço Resiliencis } & \multicolumn{2}{|c|}{ Atençio pleni Bem ettaf } & \multicolumn{2}{|c|}{ Bem etar esp felicidade } & \multicolumn{2}{|c|}{ Satistosço cor Otimismo } \\
\hline & & $6 \cdot 0,11$ & $4 \cdot 0,08$ & $1 \cdot 0,02$ & 6 & na & $1.0,02$ & 4 \\
\hline Resiliencia & $6 \cdot 0,11$ & & & $3.0,06$ & $1 \cdot 0,02$ & noa & $1 \cdot 0,03$ & va \\
\hline Atençio plens & $4.0,08$ & $5 \cdot 0,10$ & & & $2 \cdot 0,05$ & $1.0,02$ & va & nas \\
\hline Bem estar & $1.0,02$ & $3.0,06$ & & & 90 & $2 \cdot 0,05$ & $2 \cdot 0,06$ & $1.0,03$ \\
\hline Bem estar espiritual & nio & $1.0,02$ & $2 \cdot 0,05$ & $4 \cdot 0,11$ & & 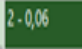 & & $n a$ \\
\hline Felicidade & nat & na & $1.0,02$ & $2.0,05$ & $2.0,06$ & & $4 \cdot 0,15$ & $2 \cdot 0,07$ \\
\hline Satistoçjo com a vida & $1.0,02$ & $1 \cdot 0,03$ & for & $2 \cdot 0,06$ & & $4 \cdot 0,15$ & & $5.0,26$ \\
\hline Otimismo & no & no & $n a$ & $1.0,03$ & a & $2 \cdot 0,07$ & $5 \cdot 0,26$ & \\
\hline
\end{tabular}

Legenda: para cada par de categorias é indicado o $\mathrm{n}^{\mathrm{o}}$ de cotações em que as categorias foram codificadas com outro código, no total ou em parte da citação e o índice de correlação (c-coeficiente) que indica a força da relação entre dois códigos.

A metodologia da análise envolve ainda a apresentação de extractos das entrevistas, apresentados por temas e subtemas revelando 


\section{Silva, Catarina, Rosado, António}

pontos em comum e diferenças entre os entrevistados, representando as ideias chave e destacando relações entre conceitos, como indicam Chandler, Anstey, e Ross (2015).

\section{Efeitos gerais da prática do Yoga}

Após as questões de caraterização geral, os participantes foram questionados sobre os efeitos em geral do Yoga nas suas vidas.

Os entrevistados sentiram necessidade de definir, em primeiro lugar, o conceito de Yoga. Yoga apresentando o significado de União, de integração consigo próprio, como ser humano e também socialmente, para viver melhor e melhorar a sociedade. Para um dos entrevistados: "O Yoga, no verdadeiro sentido, é a união espiritual. As posturas de Yoga são práticas auxiliares para esse objectivo humano último." (M5)

Em seguida, as respostas foram unânimes considerando existirem benefícios pessoais, em aspetos físicos e psíquicos, mas, particularmente ligados ao Sentir, à esfera emocional, desenvolvendo e procurando uma sensibilidade interior, um equilíbrio pessoal, com elevados níveis de espiritualidade. O Yoga é visto como tendo uma dimensão espiritual que apela à transcendência, mas não se tratando no entanto, de uma atividade religiosa. É o que referem M4 e F1: "no fundo essa felicidade, que tanto o Yoga como o budismo, nos ensinam, que essa felicidade vem de um bem-estar interno, a questão de aceitarmos como somos, de aprendermos com isso." (M4) e "Bem-estar espiritual, acho que isso é o mais importante no Yoga, mais importante que o físico, é o bemestar espiritual.Se nós tivermos um bem-estar espiritual, depois ele transmite-se e vai contagiar, digamos, a parte fisica." (F1)

As práticas realizadas no Yoga, de acordo com todos os entrevistados, têm benefícios físicos na medida em que prepararam e harmonizam o corpo e promovem, em particular, uma postura estável, possibilitando permanecer imóvel durante muito tempo, referida como condição para meditar confortavelmente: “as posturas têm uma finalidade importante que é a desintoxicação e o trabalho sobre o sistema nervoso, de forma que ele se possa estabilizar em equilibrio" (M1) e também: "o Yoga é a integração do ser completo, a parte física, a respiração, e o mental. O fim em vista, da prática do Yoga, que pode ser diversa, é no fundo atingir a superação do ser." (M2)

Se a promoção de capacidades físicas é importante, elas parecem, no entanto, terem um papel instrumental. Neste contexto, todos os entrevistados referem ser fundamental a respiração, existindo uma relação estreita entre a respiração e os estados mentais. Afirmando que: "a prática do Yoga o que dá primeiro é um equilibrio de saúde fundamental e depois uma 


\section{Efeitos psicossociais do Yoga}

transformação de atitude interior com o exterior. Existe uma vivência interior permanente que é dada através da consciência da respiração." (M1)

Segundo F3: "às vezes digo às pessoas, olhe, respire. Respirar resolve logo o problema, porque o principal remédio para a vida é a respiração." Outro entrevistado acrescenta que: "há uma ligação muito estreita entre a respiração e os estados mentais. Quando alguém está sereno, a respiração é harmónica, lenta e profunda. Quando alguém está descontrolado, exaltado, a respiração é ofegante, desarmónica, descontrolada." (M3)

Efeitos psicossociais da prática do Yoga

Relativamente aos efeitos psicossociais da prática de Yoga divide-se a apresentação dos resultados nos seguintes subtemas: bem-estar subjetivo, bem-estar espiritual, felicidade e satisfação com a vida, otimismo, atenção plena, capacidade de lidar com as adversidades, autoavaliações nucleares, autoconhecimento e identidade e efeitos psicossociais negativos.

\section{Bem-estar subjectivo}

O bem-estar subjetivo é entendido como um elevado sentimento de bem-estar, envolvendo a presença de afetos positivos e ausência de negativos. O bem-estar subjetivo é considerado como o resultado do efeito cumulativo de experiências positivas em vários domínios específicos da existência, como o trabalho, a família e o lazer (Diener, 2000). Segundo Karakashian (2011), o bem-estar apresenta duas componentes: hedónica, com a felicidade, a vivência de sentimentos agradáveis e o afecto e eudemónica com o sentido de congruência pessoal na vida, de realização pessoal.

A conceção dominante dos entrevistados refere que, com o Yoga, aprende-se a encarar as próprias limitações e a não fazer delas um problema, o que contribui para melhorar a qualidade de vida e o bem-estar. Como aponta F1: “com o Yoga nós conseguimos encarar as nossas limitações e não fazer delas um problema, mas fazer delas uma forma de qualidade de vida. Usar as limitações e transformá-las em qualidade de vida.”. O Yoga “contribui para o bem-estar porque as pessoas tomam mais consciência de si próprias, do corpo, daquilo que sentem, tomam consciência da respiração. É um passo para viver um bocadinho melhor." (M2)

Os entrevistados referem que as pessoas, ao praticar Yoga, tomam maior consciência de si próprias, do corpo, da respiração, das suas acções, daquilo que sentem e passam a realçar os aspetos positivos da vida, vendo o mundo com outros olhos, numa perspetiva eudemónica de autoconhecimento. Esse bem-estar acaba por se transmitir aos outros. Apresentam a ideia que: " $a$ proposta do Yoga visa o autoconhecimento. Um expandir de consciência que seja o 


\section{Silva, Catarina, Rosado, António}

autoconhecimento.” (M4). Reforçada com a afirmação de que "nós estamos tão bem que transmitimos essa sensação. É uma transmissão de bem-estar, em cadeia." (F5)

A melhoria que os entrevistados referem acontecer a nível do bem-estar subjetivo está de acordo com os resultados de vários estudos experimentais com populações diferenciadas (Silva e Rosado, 2017).

\section{Bem-estar espiritual}

Ligado ao bem-estar geral surgem referências ao bem-estar espiritual, que, na opinião dos entrevistados, ajuda a manter a saúde e a vitalidade. O bem-estar espiritual é definido por Gomez e Fisher (2003) como o estado do ser que reflete os sentimentos positivos, os comportamentos e a cognição das relações consigo próprio, com os outros, com o transcendente e com a natureza, prevendo um sentido de identidade, totalidade, satisfação, harmonia, propósito e direção de vida. A espiritualidade é inerente a todos os seres humanos e não deve ser confundida com nenhuma crença ou tradição religiosa, podendo influenciar a saúde física e mental (Chirico, 2016), ajudando a regular as emoções e também os efeitos fisiológicos (Aldwin, Park, Jeong, \& Nath, 2014).

É consensual, para todos os entrevistados que a prática do Yoga tranquiliza a mente proporcionando um profundo estado de relaxamento, descontracão e paz interior, com estreita ligação à espiritualidade, o que está de acordo com Ivtzan e Papatoniou (2014), Rathore, e Choudhary (2013), Bussing, Ostermann, Ludke, e Michaelsen (2013).

A orientação para o bem-estar espiritual é referida por um dos professores, indicando que propicia um sentido para o transcendente, uma experiência não necessariamente religiosa, entendendo-se como procura e focalização em valores existenciais, no significado da vida: "evolução espiritual significa relaxamento. Relaxamento e evolução espiritual são sinónimos. Quanto mais a pessoa evolui espiritualmente, quanto mais mergulha nos reinos subtis, mais relaxada fica, autoconfiante, esperançosa." (M3).

\section{Satisfação com a vida e Felicidade}

A felicidade e a satisfação com a vida surgem nas entrevistas como conceitos interligados. A felicidade pode ser considerada como o grau com que a pessoa avalia globalmente a positividade da vida, ou seja, quanto dela gosta, combinando duas componentes: afetiva, com uma forma particular de estar na vida, com despojamento material, e cognitivo, associado a uma melhor compreensão das pessoas (Lyubomiasky e Lepper, 1999), ambas as componentes referenciadas pelos entrevistados que referem que o Yoga torna os seus praticantes mais felizes. A felicidade não sendo encontrada 


\section{Efeitos psicossociais do Yoga}

no exterior, vindo de um bem-estar interno de aceitação pessoal. A proposta do Yoga visa a aceitação de si próprio, o autoconhecimento como fonte de realização pessoal e ver a vida de forma diferente, cultivar o pensamento positivo e ser, assim, mais feliz. Surgem as afirmações: “o Yoga dá-nos uma visão da felicidade muito mais ampla e menos estereotipada. Uma visão completamente ampla do que é a felicidade. Nós precisamos de pouca coisa, para sermos felizes, desde que aprendamos a ser felizes internamente." (M4): "sou muito feliz, o Yoga contribuiu muito para isso”. (F2)

$\mathrm{Na}$ opinião de dois dos entrevistados o que poderia ser considerado como uma dificuldade pode tornar-se possível através da prática de Yoga, a qualidade de vida melhora e assim a pessoa consegue ser mais feliz: "com o Yoga conseguimos encarar as nossas limitações e não fazer delas um problema, mas uma forma de qualidade de vida. Usar as limitações $e$ transformá-las em qualidade de vida. Consegui isso e por isso considero-me uma pessoa feliz." (F1). Outro entrevistado e acrescenta: " $a$ energia que nos é dada a conhecer, mantem-se em todas as situações do viver, mesmo na dor, que nos leva à sua aceitação/compreensão como parte do viver, bem como os momentos de satisfação/alegria." (M1)

Por outro lado, os professores também referem o facto de conseguirem observar a felicidade na face dos alunos durante a aula e quando esta termina. É a opinião: “a gente sente nas aulas ... a gente olha para os alunos...realmente extraordinário, vem de dentro." (F5)

Associada à felicidade surge a satisfação com a vida, que é a componente cognitiva do bem-estar (Diener, 2000), resultado de um processo de julgamento consciente, onde as pessoas avaliam a qualidade das suas vidas com base em critérios pessoais. O Yoga contribui para essa transformação da pessoa porque permite tomar consciência e estar grato por aquilo que a vida proporciona. Dizem mesmo: "há tantos anos metida nisto que acho que o Yoga me transformou na pessoa que eu sou. Uma pessoa bem-disposta, uma pessoa que está bem consigo e com os outros..." (F1) e também: "dá uma satisfação com a vida. Porquê. Porque nós não nos conhecemos, passamos a conhecer-nos melhor. O nosso conhecimento é tão diferente." (F5)

Para que aconteça a satisfação com a vida, na opinião dos professores, tem de existir um envolvimento com algum despojamento material, atingindo-se um estado de tranquilidade interna. Este estado de"satisfação não é o aumentar das posses, satisfação é tranquilização interior, é mente serena." (M3).

\section{Otimismo}

Outro construto que emerge das entrevistas é o otimismo. Uma dimensão da personalidade que 


\section{Silva, Catarina, Rosado, António}

se liga à expectativa de que as coisas vão correr bem, envolvendo uma interação entre a aceitação e compreensão positiva da realidade, com a capacidade de antecipar o futuro, aceitar a mudança e o imprevisto (Scheier, Carver, \& Bridges, 1994). O Yoga proporciona um optimismo que pode ser considerado realista. " $O$ optimismo está inerente à prática, mantem-se em todas as situações do viver...considerando toda a força que o Yoga nos proporciona, o dia-a-dia tem um outro valor, as adversidades servem de desafio, para criar um sentido positivo da vida."(M1)

O que os professores referem é que o otimismo é inerente à prática de Yoga e que a energia dada a conhecer se mantém em todas as circunstâncias, o que está de acordo com os estudos de Reis e Alliggod (2014). "A paz, a felicidade, caracterizam o ser humano sadio, sábio. Elas proporcionam o otimismo, a satisfação com a vida. Está tudo inter-relacionado. Se a pessoa é feliz, está satisfeita. É outra dádiva do Yoga." (M3)

\section{Atenção plena}

A atenção plena (mindfulness) é um estado em que a atenção se encontra focada no momento presente, aceitando-o sem julgamento e em que coexistem cinco fatores: a observação das experiências internas e externas; a descrição das experiências interiores por palavras; as ações realizadas com conhecimento; o não julgar de acordo com os pensamentos e sentimentos e não lhes reagir (Kabat-Zinn, 2013).

A atenção plena e a meditação são vistas, pelos professores experientes, como formas de regulação emocional, de maior atenção ao quotidiano, garantindo maiores níveis de autocontrolo, como caminho para concretizar os objetivos de vida. Maiores níveis de consciencialização de si e dos outros exigem níveis mais elevados de atenção aos pormenores do dia a dia evitando pensamentos automáticos. A prática de Yoga contribuindo assim, para um aumento da atenção plena.

Como referem os entrevistados praticar Yoga: "é estar atento à vida, é olhar as pequeninas coisas que a vida nos dá e que nos ensina...é um olhar diferente que o Yoga nos dá." (F5) e ainda que "a prática do Yoga é efetivamente uma chamada ao momento presente, ao aqui e agora, estar totalmente consciente. É no fundo mindfulness. A tomada de consciência de cada momento e de cada acção. Temos de estar permanentemente conscientes." (M2)

Os experts referem que durante a prática de Yoga tem de se estar no "aqui e agora", não é apenas exercício físico, mas julgar menos, sem se apegar aos estados mentais e emocionais. Os entrevistados apresentam definições de atenção plena: "o mindfulness é isso mesmo. Eu estar no presente, no momento." F1 e outro dos entrevistados: o momento presente é esse é que 


\section{Efeitos psicossociais do Yoga}

importa, o dia a dia, sem julgamento, com análise, mas sem julgamento. Procuro em cada momento estar no aqui e agora, constatando o que se está a passar sem julgar.” M2

Das entrevistas ressalta a ideia de que a prática das posturas e da meditação estão intimamente ligadas, com a chamada ao momento presente, num processo de total consciência. A pessoa, mais atenta, sente as suas energias mais equilibradas e, estando focada e concentrada, consegue encontrar soluções mais facilmente, não se dispersando. No Yoga parece ser fundamental a observação da mente, compreendendo como funciona, serenando-a, acalmando-a e tranquilizando-a. Como indica M1, adquire-se: “uma visão muito mais ampla em todos os campos da vida, podemos estar atentos às situações que vamos vivendo, $e$ controlá-las, de forma que não nos tirem do caminho, antes pelo contrário, direcioná-lo melhor". Trata-se na opinião de outro entrevistado: “viver o momento presente é um treino, o Yoga ensina-nos a estar concentrados. Duas coisas não cabem no mesmo sítio ao mesmo tempo. Com o pensamento é igual." (F3) Capacidade de lidar com as adversidades

A resiliência pode ser definida como a capacidade de o indivíduo se adaptar ultrapassando situações adversas, não apenas de carater individual, podendo variar com as circunstâncias e com o tempo (Rutter, 1999). É um processo dinâmico, complexo, em que as pessoas interagem e são influenciadas pelos contextos físicos, e sociais, existindo uma variação que é individual e que pode variar face à situação e ao próprio indivíduo (Wagnild e Young, 1993).

Quando questionados relativamente ao modo como o Yoga ajuda a lidar com as adversidades que a vida vai colocando, os entrevistados referem que prática promove a capacidade de adaptação às mudanças, em que a pessoa adquire mais flexibilidade para contornar obstáculos.

A opinião dos entrevistados segue as indicações de Telles, Singh, Bhardwaj, Kumer, e Baikrishna (2013), considerando que a prática de Yoga contribui para aumentar a resiliência dos indivíduos. Apontam que: "o Yoga consegue ajudar as pessoas a resolver problemas, desde que a pessoa queira, que pense que pode fazê-lo. O Yoga dá-lhe as ferramentas.” (F1). Acrescentam que: "quem pratica Yoga adquire a capacidade de enfrentar as adversidades corajosa e serenamente; de fazer frente às situações mais problemáticas; ao mesmo tempo adquire a consciência de que tudo passa, tudo é passageiro e não digno de preocupação excessiva." (M3)

O Yoga é visto como um processo de autosuperação. Mesmo quando existem experiências traumáticas anteriores, pode ajudar a enfrentálas, dando uma dimensão mais ampla a todos os 


\section{Silva, Catarina, Rosado, António}

campos da vida, com mais atenção às situações, controlando-as melhor e com maior capacidade de resolução de problemas. Para os entrevistados "mesmo as influências traumáticas do passado, que possam surgir, neste jogo mental, o Yoga ajuda a enfrentá-las, e dissipa-las." (M1). Outro entrevistado refere que: "O Yoga ajuda a lidar com problemas, para já nós estamos mais preparados fisicamente e também o nosso cérebro está mais fortalecido." (F5)

A prática de Yoga é vista como oferecendo mais confiança à pessoa, mais entusiasmo e essas características passam para a vida. O que é exemplificado por "e depois dá mais confiança à pessoa, mais força de vontade, mais entusiasmo. Passa da aula de Yoga para o exterior.” (M2), e ainda: “a mente não vá atrás das atitudes, das influências que possam haver no dia a dia." (M1). Outro entrevistado acrescenta: "uma preparação melhor que o Yoga nos dá, o sofrimento também não quer dizer que não exista mas é visto de uma maneira diferente." (F5)

É unânime, para os entrevistados, que o Yoga ajuda a pessoa a ficar fortalecida mentalmente. $\mathrm{O}$ equilíbrio conseguido mantém-se em múltiplas situações, mesmo menos positivas, levando a uma maior aceitação e compreensão dos acontecimentos. As posturas contribuem para um "sistema nervoso forte, mente sã : as posturas do Yoga vão actuar directamente no SNC, dando uma fortaleza pacífica ao corpo, e este à mente." (M1) alterando mesmo "a maneira de pensar em situações mais complicadas ... a grande vantagem do Yoga é aumentar a nossa energia, para que quando houver um desafio, contrariedade, nós temos um extra de energia, para enfrentar." (M4)

\section{Autoavaliações nucleares}

Outra das caraterísticas da personalidade que pode levar a um aumento do bem-estar são as autoavaliações nucleares que refletem as avaliações que os indivíduos fazem de si próprios, nomeadamente, as crenças de autoeficácia, o locus de controlo, a autoestima e a estabilidade emocional (Judge, Erez, Bono \& Thorensen, 2003), sendo indicadas como afetadas positivamente pela prática de Yoga (Rissel, Miller, Lloyd, \& Williams, 2014). Os entrevistados referem-se a estas avaliações: “depois dá mais confiança à pessoa, mais força de vontade, mais entusiasmo. Passa da aula de Yoga para o exterior." (M2) e também que "a prática do Yoga gera autoconfiança. A autoconfiança, a fé em si mesmo, são geradas pela prática." (M3)

O locus de controlo pode ser entendido como a forma como o indivíduo perceciona as variáveis que controlam os acontecimentos da sua vida; um locus interno significa que o indivíduo acredita que um acontecimento está sob o seu controlo e o externo quando acredita que o 


\section{Efeitos psicossociais do Yoga}

ambiente ou o destino controlam os acontecimentos. Os entrevistados consideram que, com a prática regular, ao estar atento, de forma continuada, à consciencialização dos movimentos e respirações, dá-se uma responsabilização individual que fundamenta a perceção de controlo, garantindo formas de maior autocontrolo interno. Estas afirmações podem ser ilustradas: "todas as situações são controladas...não um controlo, mas uma consciencialização." (F4) e “ajudou-me bastante, no fundo em controlar um bocadinho melhor as emoções, as minhas reacções, a melhorar o equilíbrio energético, o corpo." (M2)

A estabilidade emocional é potencializada com a prática do Yoga, segundo opinião dos entrevistados. $\mathrm{O}$ controlo da respiração permite atingir um estado de calma que leva a gerir melhor as emoções. Também o desapego das coisas e o distanciamento das situações, traz uma paz interior, harmonia, ajudando a controlar melhor as emoções. A prática de Yoga gera não só autoconhecimento, mas também autoconfiança, o acreditar em si mesmo, diminuindo a ansiedade. Estabilidade emocional que pode ser percebida nas seguintes asserções expressas: "o Yoga ajudou-me bastante, a controlar um bocadinho melhor as emoções, as minhas reacções, a melhorar o equilibrio energético, o corpo." (M2) e, ainda, para outro entrevistado "estabilidade emocional muita...é aquela paz, aquela harmonia, um estado que nós também temos de o cultivar, temos de o aprender. É o desapego, o desapego de coisas, de situações familiares, vai trazendo essa paz interior." (F5)

\section{Autoconhecimento}

O autoconhecimento expressa o conhecimento sobre si próprio, em que se consegue diferenciar entre comportamento próprio e as variáveis que o controlam. Um melhor conhecimento de si próprio pode contribuir para um aumento do bem-estar. A prática de Yoga é considerada, por todos os participantes, como contribuindo para se conhecer melhor, perceber quem se é realmente, com uma melhor aceitação das suas forças e fraquezas, potencializando a autoestima. As afirmações seguintes ilustram estas ideias: "passamos a conhecer-nos melhor ...uma alegria de viver." (F5) e, “o Yoga pode influenciar tudo o que você faça no seu dia a dia, tudo, quer seja no seu trabalho, quer seja nos seus hobbies, quer seja numa prática de desporto. Pode influenciar em tudo." (F1)

A identidade pessoal, entendida como resultado da dinâmica entre a história pessoal, o contexto histórico, e social e os projetos de vida, é influenciada com a prática do Yoga, segundo os entrevistados. Como referem dois dos entrevistados: "eu estou há tantos anos metida nisto que acho que o Yoga me transformou na 


\section{Silva, Catarina, Rosado, António}

pessoa que eu sou. Uma pessoa bem disposta, que está bem consigo e com os outros. O Yoga está em tudo na vida." (F3) e, "praticarmos Yoga exige uma mudança de determinados padrões. A pessoa descobre a integração da consciência e do corpo e começa a perceber que há factores que tem de mudar, principalmente a alimentação, postura, relacionamento com os outros." (M2)

\section{Efeitos negativos}

O Yoga também é percebido, pelos professores, como podendo potenciar efeitos negativos, quer a nível físico, quer psicossocial. A nível físico, os entrevistados referem a possibilidade das posturas serem realizadas de forma incorreta, se os praticantes não tiverem consciência do seu corpo, se exagerarem ou forçarem, podendo mesmo lesionar-se. Também se existirem problemas de saúde específicos (como por exemplo: hipertensão), devem evitar-se algumas posturas ou fazer determinadas posturas de forma controlada. Aparecem nas palavras dos entrevistados: "O Yoga não tem só coisas boas, não quer dizer perigosas, mas efeitos maus se a pessoa não tiver consciência do seu corpo na prática de asanas pode ter muitos efeitos maus, lesões várias e eu conheço." (M1). Podendo mesmo, na opinão deste professor, ser: "prejudicial se a pessoa começar a entrar em competição até consigo própria”. (M1) Para outro entrevistado "pode causar problemas físicos e além disso, o professor que está à frente disso tem de ter o discernimento de saber o que é que pode fazer." (F2) e confirmado: "o problema é quando uma pessoa pratica yoga pensando que está a praticar outro tipo de actividade física qualquer. O Yoga é uma filosofia de vida, uma maneira de estar na vida, que é de respeitar o seu ser." (M2)

A nível emocional e espiritual a prática de Yoga também pode apresentar consequências negativas. O que é explicado por um dos entrevistados: "quando estão a descontrair vem ao de cima, as memórias e as memórias nem sempre são aquela coisa fantástica." (F3) e ainda outro esclarecimento: "pode haver problemas de pessoas que estão a nível psicológico a serem muito afectadas. E muito afectadas, depende da pessoa que estiver à frente." (F1)

Numa outra abordagem, os entrevistados referem que a individualidade do professor, o culto da personalidade e o radicalismo, são ameaças potenciais aos praticantes, com a existência de grupos fechados de praticantes de Yoga, de tendência fundamentalista presente no discurso e nas práticas. Seguem-se algumas citações: "pode haver problemas, uma pessoa se tem uma mente muito forte, e que dominam pode influenciar os outros...Mandar nos outros, isso é que é o perigo." (F2), reforçadas por outro entrevistado: "há professores que são extremamente duros 


\section{Efeitos psicossociais do Yoga}

com os alunos. Há seitas porque o Yoga serve para apoiar e criar cultos de personalidade." (M1) e, ainda:"as técnicas de Yoga são poderosíssimas e de efeitos calamitosos se "ensinadas" por ignorantes, por meros curiosos.” (M3)

Um dos professores coloca mesmo em causa a designação de algumas práticas lecionadas como sendo de Yoga, na sua opinião, oferecem risco: “as pessoas não estão a aprender Yoga coisa nenhuma, porque as bases do Yoga não lhes foram transmitidas, é um grande risco.” $\mathrm{F} 1$

Outro dos professores considera que surgem diversas denominações para designar as práticas de Yoga, mas: "agora há muitas modalidades de Yoga, em cima da prancha, no paddel, são variantes ocidentalizadas, acho que não fazem mal nenhum, só ajudam a difundir o Yoga, portanto não tem problema nenhum." (M2). O problema para este entrevistado surge quando: "há os radicalismos, mas isso há em todo o lado."

Para um dos professores, o Yoga não é uma prática adequada a todas as pessoas: "o Yoga não é para toda a gente. Exige de tal maneira em termos de sentir, que é muito poderoso. Nem todas a pessoa têm capacidade nem têm estrutura." (F3) Este professor explica que, para evitar problemas: "eu gosto de oferecer a $1^{a}$ aula, para as pessoas verem se se sentem bem aqui, com este método de trabalho ou se querem outra coisa."

Para um dos entrevistados não existem efeitos negativos na prática de Yoga, o Yoga não se distingue de uma outra prática: "não prejudica absolutamente nada. Tanto a nível físico como dos nossos órgãos, que são beneficiados com as práticas... é assim em todos os desportos, se a pessoa exagerar, ou forçar, não pensar no que está a fazer, também sofre." (F5)

Outro entrevistado concorda em que não existem efeitos negativos desde que se encontrem presentes no professor algumas caraterísticas: "quando o professor de Yoga é competente, dotado de grande experiência e nobreza de caráter, não haverá, na prática do Yoga, efeitos colaterais indesejáveis. Só benefícios acontecerão." (M3). Indica que poderão existir limitações em caso de algumas doenças como exemplifica: "se for um aluno que tenha, por exemplo, esquizofrenia, nunca deve ir para uma aula de Yoga.... Visualizações estranhas, em que esse aluno vai começar a ter, mas porque não está bem, porque é doente." (F5)

Representação visual dos dados das entrevistas A partir das transcrições escritas das entrevistas, com a contagem de palavras realizada pelo programa ATLAS.ti 7, foi feita uma nuvem de palavras com as palavras referidas nas várias entrevists. $\mathrm{O}$ tamanho visual das palavras indica a sua frequência na totalidade das entrevistas 


\section{Silva, Catarina, Rosado, António}

transcritas (WordArt). A imagem visual em nuvem permite representar visualmente as palavras dos entrevistados, ajudar a capturar a essência dos seus discursos, dando-lhes significado, reduzindo e focando-as palavras (Chandler, Anstey, e Ross, 2015), permitindo encontrar padrões e valores extremos, introduzindo novos níveis de entendimento a partir das palavras dos entrevistados (Figura 2).

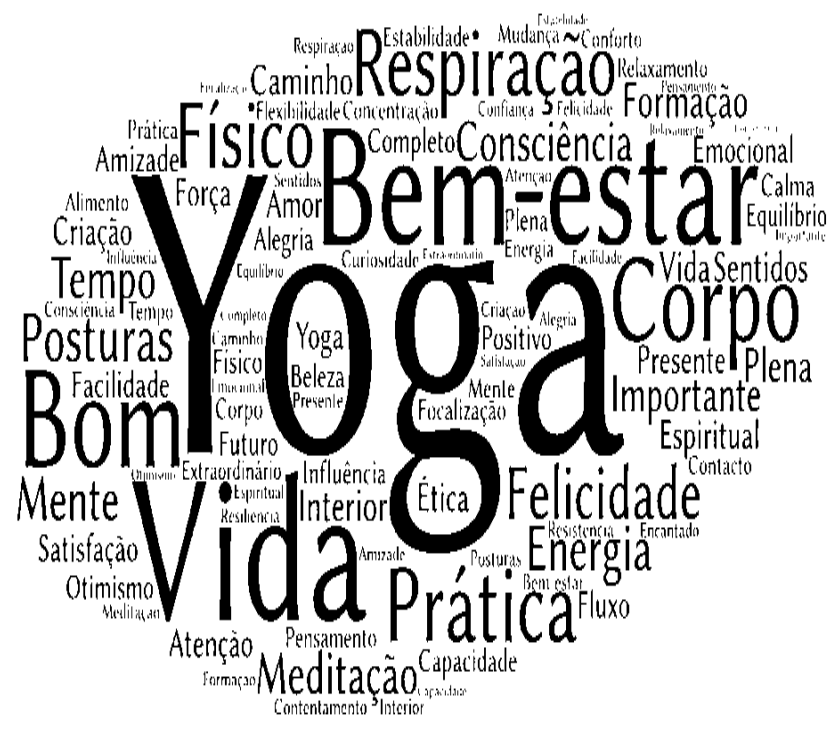

Figura 2. Nuvem de palavras o Yoga e os efeitos psicossociais: palavras dos professores experientes.

\section{DISCUSSÃO}

Os dados recolhidos descrevem como professores pioneiros e com longa experiência de lecionação em Yoga em Portugal, considerados experts nesta atividade, percebem os efeitos psicossociais da prática de Yoga. A análise dos dados aponta para que todos os professores consideram que a prática de Yoga tem efeitos psicossociais positivos, nomeadamente, a nível da melhoria do bem-estar físico e social, do bemestar espiritual, do otimismo, da satisfação com a vida, da felicidade, da resiliência, da atenção plena e das autoavaliações nucleares.

Estes entrevistados permitiram uma riqueza de informação porque se tratam de pessoas com uma grande experiência na área abordada, com um conhecimento importante de preservar. De acordo com as suas experiências, e com o que observaram nos seus alunos, todos consideram que o Yoga teve um forte impacto nas suas vidas, influenciando-as e contribui para um aumento do bem-estar, o que confirma os dados obtidos na revisão sistemática de Silva e Rosado (2017).

Verifica-se que, em particular para os professores com mais idade, mais tempo de prática e de lecionação, o Yoga se confunde com a sua própria vida, não conseguindo definir a sua vida sem Yoga. Estes professores com mais idade, e mais tempo de prática e de lecionação construíram a sua identidade pessoal em torno desta vivência. Tal como descrevem, as suas vidas transformaram-se, o Yoga influenciou tudo o que fazem, sublinhando que a prática se orienta para a transformação da pessoa; é um projeto assente numa filosofia de vida, numa espiritualidade, propondo uma identidade pessoal e cultural, uma determinada forma particular de estar, um estilo de vida único. 


\section{Efeitos psicossociais do Yoga}

\section{CONCLUSÕES}

Embora se tenha verificado uma imagem bastante positiva em relação à relevância de vários efeitos positivos psicossociais, tal conclusão só deve ser vista como uma tentativa. Além disso, as entrevistas, por vezes, podem ser vistas como totalmente dominadas pelo entrevistador, que restringe as respostas dos entrevistados, comprometendo a profundidade da investigação. Por isso, é necessário fazer um trabalho empírico (e mais importante) ainda mais antes de fazer preposições mais sólidas; Isto é particularmente assim em termos de provocar o que os professores de Yoga consideram importantes em termos de priorização das atividades diárias que promovem cada efeito psicossocial.

\section{APLICAÇÕES PRÁTICAS}

Este estudo reúne um conjunto de argumentos, neste caso baseado em experiências pessoais de professores muito experientes, que sustentam a legitimação do Yoga como uma prática física com efeitos positivos sobre aspectos essenciais do desenvolvimento psicossocial dos praticantes. Essa legitimação terá que ser baseada na investigação científica e a recomendação para essa prática assente num claro entendimento acerca dos seus benefícios e malefícios potenciais. Os relatos subjectivos, como neste caso, a distilação das experiências de vida dos professores experts de Yoga, juntamente com as análises sistemáticas da literatura científica sobre os efeitos psicossociais do Yoga devem ajudar a legitimar essas práticas. O estudo da eficácia e da utilidade destas práticas sai beneficiada e um melhor serviço poderá ser prestado aos praticantes. A eficácia diz respeito à força das evidências de relações causais entre a prática do yoga e os resultados desta sobre um efeito psicossocial específico. Essa investigação deve continuar. Evidentemente importa equacionar, também, a questão da perícia ou da competência dos profissionais para conduzirem esses processos, considerando, simultaneamente, o seu ajustamento as caraterísticas dos praticantes (diferenças individuais, momentos desenvolvimentais, etc.). A busca por evidências dos efeitos da prática do Yoga pode ser continuada lançando mão de diversos métodos de pesquisa. Estudos de caso sistemáticos, pesquisas qualitativas, delineamentos experimentais de caso único, pesquisas etnográficas, estudos longitudinais, estudos em contexto naturalístico, estudos com grupos, ensaios clínicos randomizados (randomized controlled trials na literatura, ou ECTs), metaanálises da literatura, dentre outros métodos, são complementares para compreender da forma mais abrangente possível os efeitos psicossocias 


\section{Silva, Catarina, Rosado, António}

dessas práticas. Este estudo conclui que, na opinião de professores experts de Yoga, esta tem efeitos psicossociais muito significativos nos seus praticantes. Finalmente, a prática de Yoga não é vista como potencialmente nefasta, mas quando mal orientada pode ter esse efeito. Sendo a qualidade da formação de professores e a sua experiência fundamental para garantir a minimização dos riscos, recomenda-se que os processos de formação destes profissionais possam incluir módulos sobre a legitimação científica destas práticas, esclarecendo-se o seu valor do ponto de vista dos seus reais benefícios no plano psicossocial, considerando-se planos de formação que possam optimizar esses potenciais benefícios.

\section{REFERÊNCIAS}

1. Aldwin, C. M., Park, C. L., Jeong, Y. J., \& Nath, R. (2014). Differing pathways between religiousness, spirituality, and health: A self-regulation perspective. Psychology of Religion and Spirituality, 6(1), 9-21. doi:10.1037/a0034416

2. Amado, J., Costa, A. P., e Crusoé, N. (2014). A técnica de análise de conteúdo. In J. Amado (Ed.), Manual de investigação qualitativa em educação (pp. 301-350, 2. ${ }^{\text {a }}$ ed.). Coimbra: Imprensa da Universidade de Coimbra. doi:10.14195/978-989-26-0879-2
3. Anguera, M., Portell, M., ChacónMoscoso, S., \& Sanduvete-Chaves, S. (2018). Indirect observation in everyday contexts: Concepts and methodological guidelines within a mixed methods framework. Frontiers in Psychology, 9(13). doi: 10.3389/fpsyg.2018.00013

4. Apaccarotella, L. (2017). La labor del psicólogo del deporte com la selección Argentina de Handball feminino en los Juegos Olimpicos de Brasil 2016. Revista de psicologia aplicada al deporte y al Ejercicio Físico, 1(2), 1-6. doi:10593/rpadef2017a2

5. Bilderbeck, A., Farias, M., Brazil, I., Jakobowitz, S., \& Wikholm, C. (2013). Participation in a 10-week course of Yoga improves behavioral control and decreases psychological distress in a prison population. Journal of Psychiatric Research, 47, 1438-1445. doi:10.1016/j.psychires.2013.06.014

6. Braun, V., \& Clarke, V. (2006). Using thematic analysis in psychology. Qualitative Research in Psychology, 3(2), 77-101. doi:10.1191 1478088706qp0630a

7. Bussing, A., Osterman, T., Ludtke, R., \& Michalsen, A. (2013). Effects of Yoga interventions on pain-associated disability: A meta-analysis. The Journal 


\section{Efeitos psicossociais do Yoga}

of Pain, 13(1), 1-9. doi: 10.1155 /2012/165410

8. Casaban, J., Boix V., Delcampo, D, y Chirivella, E. (2017). Intervención psicológica desde el coaching motivacional utilizando el modelo "La Girafa de Cantón" en una juegadora de futebol sala. Revista de Psicologia Aplicada al Deporte y al Ejercicio Físico, 2(10), 1-14. doi:10593/rpadef2017a12

9. Chandler, R., Anstey, E., \& Ross, H. (2015). Listening to voices and visualizing data in qualitative research: Hyper modal dissemination possibilities. Sage Open, 2(1), 1-6. doi:10.1177/2158244015592166

10. Chirico, F. (2016). Spiritual well-being in the $21^{\text {st }}$ century: It's time to review the current WHS's health definition. Journal of Health and Social Science, 1(1), 11-16. doi:10.1924/2016/sprt2

11. Diener, E. (2000). Subjective well-being: The science of happiness, and a proposal for a national index. American Psychology, 55, 34-43. doi:10.1037/0003066X.55.1.34

12. Ericsson, K., Krampe, R., \& TeschRomer, C. (1993). The role of deliberate practice in the acquisition of expert performance. Psychological Review, 100(3), 363-406. doi:0033-295X/93/S3.00
13. Friese, S. (2013). ATLAS.ti 7: User guide and reference. Berlin, Germany: Scientific Software Development GmbH.

14. Gomez, K., \& Fisher, J. W. (2003). Domains of spiritual well-being and development and validation of the spiritual well-being questionnaire. Personality and Individual Differences, 35(8), 1975-1991. doi:10.1016/SO1918869(03)00045-X

15. Gouveia, M., Pais-Ribeiro, J., Marques, M., \& Carvalho, C. (2012). Validity and reability of the Portuguese version of the Dispositional Flow Scale-2 in Exercise. Revista de Psicologia del Deporte, 21(1), 81-88.

16. Groessi, E., Chopra, D., \& Mills, P. (2015). An overview of Yoga research for health and well-being. Yoga \& Physical Therapy, 4. doi:10.4172/21577595.1000210

17. Halsall, T., Werthner, P., \& Forneris, T. (2015). Cultivating focus: Insights from dedicated Yoga practice and the implications for mental and well-being. Qualitative research in sport, exercise and health, 8(2), 1-15. doi: 10.1080/2159676X.2015.1099562

18. Ivtzan, I., \& Papantoniou, A. (2014). Yoga meets positive psychology: examining the integration of hedonic 


\section{Silva, Catarina, Rosado, António}

(gratitude) and eudemonic (meaning) wellbeing in relation to the extent of Yoga practice. Journal of Bodywork \& Movement Therapies, 18, 183-189. doi:10. 1016/j.jbmt.2013.11.005

19. Judge, T., Erez, A., Bono, J., \& Thorensen, C. (2003). The Core SelfEvaluations Scale: Development of a measure. Personnel Psychology, 56, 303$331 . \quad$ doi:10.1111/j.17446570.2003.tb00152.x

20. Kabat-Zinn, J. (2013). Full catastrophe living: Using the wisdom of your body and mind to face stress, pain (Rev. ed.). New York, NY: Delta Trade Paperback/ Bantam Dell.

21. Karakashian, M. (2011). The predictive utility of mindfulness, experiential avoidance, and values: Based action for well-being in college students. Memphis, TN: University of Memphis. doi:10 $.1007 / 978-981-10-2781-9$

22. Lincoln, Y., \& Guba, E. (1985). Naturalistic Inquiry, Beverly Hills, CA: Sage.

23. Lyubomirsky, S., Lepper, H. (1999). A measure of subjective happiness: preliminary rehability and construct validation. Social Indicators Research, 46, 137-155. doi:10.1023/A:1006824100041
24. Maxwell, J. (2013). Qualitative researches design an interactive approach (3rd ed.). London, United Kingdom: Sage Publications.

25. Meadows, L. M., \& Morse, J. M. (2001). Constructing evidence within the qualitative project. In J. M. Morse, J. M. Swanson, \& A. J. Kuzel (Eds.), The nature of qualitative evidence (pp. 187200). Thousand Oaks, CA: Sage. doi:10.4135/9781412986236.n8

26. Molina, P., Villamón, M., y Úbeda, J. (2015). La investigación cualitativa en las revistas españolas de ciencias del deporte (2007-2011). Revista de Psicologia del Deporte, 24(1), 29-36.

27. Moliver, N. M., Mika, E. M., Chartrand, M. S., Haussman, R. E., \& Khalsa, S. B. (2013). Yoga experience as a predictor of psychological wellness in women over 45 years. International Journal Yoga, 6(1), 11-19. doi:10.41030973-613.105937

28. Patton, M. (2002). Qualitative research \& evaluation methods (3rd ed). Thousand Oaks, CA: Sage.

29. Pérez-Lopez, R., Morales-Sanchez, V., Angera, M., \& Hernandez-Mendo, A. (2015). Hacia la calidad de servicio emocional en organizaciones deportivas orientadas a la población infantil: um análisis cualitativo. Revista 


\section{Efeitos psicossociais do Yoga}

Iberoamericana de Psicologia del Ejercicio y el Deporte, 10, 243-250.

30. Pérez-Lopez, R., Morales-Sanchez, V., Angera, M. \& Hernandez-Mendo, y A. (2015). Evaluación de la calidad total en servicios municipales deportivos orientados a la población infantil: Aportaciones desde el análisis cualitativo con ATLAS.ti. Cuadernos de psicologia del Deporte, 15(1), 143-150. doi:10.4321 /S1578-84232015000100014

31. Pérez-Lopez, R., Morales-Sanchez, V., Angera, M. \& Hernandez-Mendo, A. (2016). Modelo Tridimensional de la calidad en organizaciones deportivas: calidad emocional en usuários/as infatiles. Cuadernos de Psicologia del Deporte, 16(1), 243-250.

32. Rathore, N. C., \& Choudhary, M. K. (2013). Impact of Yoga on emotional intelligence and subjective well-being: a pre and post analysis. Indian Streams Research Journal, 3(7), 1. doi:10.9780/22307850.

33. Reis, P. A., \& Alligood, M. R. (2014). Prenatal yoga in late pregnancy and optimism, power, and well-being. Nursing Science Quarterly, 27(1), 30-36. doi:10.1177/0894318413509706

34. Rissel, A. A., Miller, B. K., Lloyd, L. K., \& Williams, J. S. (2014). Hot, sweaty and satisfied: Effects of Bikram Yoga on psychological well-being. Journal of Behavioral Health, 3(1), 71-76. doi:10.5455/jbh.20131231051 431

35. Saunders, B., Sim, J., Kingstone, T., Baker, S., Waterfield, J., Bartlam, B., Burroughs, H., \& Jinks, C. (2017). Saturation in qualitative research: Exploring its conceptualization and operationalization. Qualitaty and Quantity International Journal of Methodology, 52(4), 1893-1907. doi:10.1007/s1135-017 $-0574-8$

36. Scheier, M., Carver, C., \& Bridges, M. (1994). Distinguishing optimism from neuroticism (and trait anxiety, selfmastery, and self-esteem): A reevaluation of the Life Orientation Test. Journal of Personality and Social Psychology, 67(6), 1063-1078. doi/10.1037/00223514.67.6.1063

37. Silva, C. P, e Rosado, A. B. (2017). Efeitos psicossociais da prática de Yoga. Revista Iberoamericana de Psicologia del Ejercicio y el Deporte, 12(2), 203-216. Disponível em http://redalyc.org/articulo .oa?id=31115124200

38. Taspinar, B., Aslan, U., Agbuga, B., \& Taspinar, F. (2014). A comparison of the effects of Hatha Yoga and resistance on mental health and well-being in sedentary 
adults: A pilot study. Complementary Therapies in Medicine, 22, 433-440. doi:10.1016/j.ctim.2014.03.007

39. Telles, S. S., Singh, N., Bhardwaj, A. K., Kumar, A., \& Baikrishna, A. (2013). Effect of Yoga or physical exercise on physical, cognitive and emotional measures in children: Randomized controlled trial. Child and Adolescent Psychiatry and Mental Health, 20(3), 737. doi:10.1186/1753-2000-7-37

40. Wagnild, G. M., \& Young, H. (1993). Development and psychometric evaluation of the Resilience Scale. Journal of Nursing Measurement, 1(2), 165-178. 